\title{
Nipah virus persists in the brains of nonhuman primate survivors
}

\author{
Jun Liu, ${ }^{1}$ Kayla M. Coffin, ${ }^{1}$ Sara C. Johnston, ${ }^{1}$ April M. Babka, ${ }^{1}$ Todd M. Bell,, Simon Y. Long, ${ }^{1}$ \\ Anna N. Honko, ${ }^{1}$ Jens H. Kuhn, ${ }^{2}$ and Xiankun Zeng ${ }^{1}$ \\ 'United States Army Medical Research Institute of Infectious Diseases, Fort Detrick, Frederick, Maryland, USA. \\ ${ }^{2}$ Integrated Research Facility at Fort Detrick, National Institute of Allergy and Infectious Diseases, NIH, Fort Detrick, \\ Frederick, Maryland, USA.
}

\begin{abstract}
Nipah virus (NiV) is an emerging zoonotic paramyxovirus that causes highly lethal henipavirus encephalitis in humans. Survivors develop various neurologic sequelae, including late-onset and relapsing encephalitis, several months up to several years following initial infection. However, the underlying pathology and disease mechanisms of persistent neurologic complications remain unknown. Here, we demonstrate persistent NiV infection in the brains of grivets that survived experimental exposure to NiV. Encephalitis affected the entire brains, with the majority of NiV detected in the neurons and microglia of the brainstems, cerebral cortices, and cerebella. We identified the vascular endothelium in the brain as an initial target of NiV infection during the acute phase of disease, indicating a primary path of entry for NiV into the brain. Notably, we were unable to detect NiV anywhere else except the brains in the examined survivors. Our findings indicate that late-onset and relapsing encephalitis of NiV in human survivors may be due to viral persistence in the brain and shed light on the pathogenesis of chronic henipavirus encephalitis.
\end{abstract}

Conflict of interest: The authors have declared that no conflict of interests exists.

Copyright: (c) 2019, American Society for Clinical Investigation.

Submitted: April 17, 2019

Accepted: June 13, 2019

Published: July 25, 2019.

Reference information: JCl Insight. 2019;4(14):e129629. https://doi. org/10.1172/jci.insight.129629.

\section{Introduction}

Nipah virus (NiV) is a negative-sense RNA virus classified in the genus Henipavirus (Mononegavirales: Paramyxoviridae) (1). In humans, NiV causes henipavirus encephalitis (International Classification of Diseases revision 11 code 1D63) (2), a severe encephalitic disease with or without respiratory signs (3). NiV was first identified during a large human disease outbreak in Malaysia in 1998-1999 with a case fatality rate of approximately 40\% (107 deaths among 265 recorded cases). The mean duration of illness from onset of symptoms to death was 16 days (4). During that initial outbreak, direct contact with domestic pigs (Sus scrofa domesticus Erxleben 1777) was determined to be a risk factor, and domestic pigs were identified as intermediate NiV hosts. Subsequent studies revealed that domestic pigs acquired the infection from pteropodid bats that serve as natural NiV reservoir hosts $(5,6)$. Following the Malaysian outbreak, sporadic henipavirus encephalitis outbreaks have occurred almost annually in Bangladesh or India with case fatality rates averaging approximately $70 \%$ (as high as $100 \%$ in small, isolated outbreaks) $(7,8)$. In the latest outbreak in Kozhikode District, Kerala State, India, in May 2018, 21 deaths among 23 cases (case fatality rate: $91 \%$ ) were reported (9). A major route of transmission is ingestion of date palm sap contaminated by NiV-infected bats with subsequent nosocomial or intrafamilial spread (10-13).

Pathologic lesions caused by NiV infection occur in the CNS and the respiratory, vascular, and immune systems in both humans and experimentally inoculated animals, including aged and IFNAR-knockout laboratory mice, golden hamsters (Mesocricetus auratus Waterhouse 1839), domestic ferrets (Mustela putorius furo Linnaeus 1758), domestic cats (Felis catus Linnaeus 1758), domesticated guinea pigs (Cavia porcellus Linnaeus 1758), domestic pigs, and cercopithecine primates (14-27). The majority of human survivors develop neurologic sequelae, including behavioral and neuropsychiatric aberrations $(15,28)$. Relapsing encephalitis occurred in some survivors who recovered from acute encephalitis. Other survivors who were initially asymptomatic experienced late-onset encephalitis several months up to 11 years following initial infection and acute encephalitis in the Malaysian outbreak $(15,28)$. The case fatality rate of relapsed and late-onset Nipah encephalitis in humans is estimated to be approximately $18 \%$ (28).

Nonhuman primates (NHPs) have been used as a model for henipavirus encephalitis because NHPs develop disease that is strikingly similar to human disease (20). The occurrence of relapsing and late-onset 
encephalitis of $\mathrm{NiV}$ in human survivors (28) prompted us to investigate persistent $\mathrm{NiV}$ infection in the brains of NHP survivors. We identified the cellular reservoir of NiV persistence in the brain of grivet (Chlorocebus aethiops Linnaeus 1758) survivors with severe encephalitis and the initial process leading to such persistence.

\section{Results}

NiV persists in the brains of grivet survivors. The occurrence of relapsing and late-onset encephalitis of NiV in human survivors (28) prompted us to investigate persistent NiV infection in the brains of NHP survivors. The brain is also a well-recognized immune-privileged organ similar to the eye and testis. We investigated the potential for persistence of NiV infection of NHP survivors.

Two grivet survivors were identified from a previous study in which 4 grivets were exposed intratracheally (i.t.) at a Biosafety Level 4 laboratory with $2.5 \times 10^{4} \mathrm{PFU}$ of the Malaysian strain of NiV (21). These 2 animals had disease of varying severity, including axillary lymphadenopathy, elevated body temperature, decreased responsiveness, and labored breathing, but they survived and were euthanized on day 32 after exposure (scheduled end of study) without any treatment (21). Using RNA ISH, we detected NiV genomic RNA only in the brains of both survivors, but not in any other organs evaluated, including colon, eyes, liver, lungs, lymph nodes, spleen, stomach, and testes (Supplemental Table 1; supplemental material available online with this article; https://doi.org/10.1172/jci. insight.129629DS1). Consistently, viral genomic RNA was not detected in PBMCs of either survivor by quantitative reverse transcription PCR (RT-qPCR) at the end of the study (Figure 1A) (21). NiV RNA was detected mainly in the brainstem, cerebral cortex, and cerebellum (Figure 1, B-E, and Supplemental Table 1). Furthermore, NiV antigenomic RNA (positive-sense RNA) was detected by multiplex fluorescence ISH (mFISH) in the brains (Figure 1, F and G), and NiV antigen was detected again in the brainstem, cerebral cortex, and cerebellum by IHC (Figure 1, H-J). Together, the detection of NiV genomic RNA and antigenomic RNA and antigen indicate active NiV replication in the brains of grivet survivors of previous NiV infection.

Severe encephalitis is a hallmark of grivet survivors persistently infected with NiV. Tissues from different brain areas of both survivors were collected and stained with H\&E for histopathology analysis. The key histopathologic brain lesions were severe encephalitis and vasculitis in the cerebrum and cerebellum with or without meningitis (Figure 2, A-F). Notably, encephalitis was prominent in the brainstem and cerebellum (Figure 2, D-F). The encephalitis in these cases consisted of lymphohistiocytic inflammation and varying degrees of necrosis, rarefaction, spongiosis, and microgliosis (Figure 2, A-F). Compared with uninfected control grivet brain tissue, proliferating $\mathrm{Ki} 7^{+}$cells, infiltration of predominantly $\mathrm{CD} 68^{+}$microglia (resident macrophages; Figure 2, G and $\mathrm{H}$ ), and some infiltration of $\mathrm{IgG}^{+}$cells and $\mathrm{CD} 20^{+} \mathrm{B}$ cells (Figure 2, I and $\mathrm{J}$ ) suggest that survivor brains may no longer serve as immune-privileged sites.

NiV persists mainly in brain neurons and microglia. To identify the cellular targets of persistent NiV in survivor brains, we performed immunofluorescence staining using one Ab against cell-specific markers and a second $\mathrm{Ab}$ against NiV. Consistent with ISH and IHC results, NiV antigen was multifocally detected by immunofluorescence. Interestingly, NiV antigen was detected mainly in neurons labeled by an anti-NeuN Ab, an established marker for neurons (Figure 3A), among NiV antigen-positive cells. Also, NiV antigen could be detected in microglial cells (macrophages) that express CD68 (Figure $3 \mathrm{~B})$. These data indicate that neurons and microglia are the main cellular targets of persistent NiV in survivor brains.

$\mathrm{NiV}$ brain persistence is established through infection of the vascular endothelium. We analyzed brain tissues from 6 grivets that had pneumonia and gliosis and succumbed to acute NiV disease between day 9 and 11 after i.t. exposure to $2.5 \times 10^{4}$ or $2 \times 10^{5} \mathrm{PFU}$ of NiV (unpublished observations); Supplemental Table 2). Strikingly, the NiV infection pattern in the brain was highly consistent in all 6 grivets. NiV genomic RNA and/or antigen were detected mainly in the endothelial cells of some vessels of both cerebra and cerebella (Figure 4, A and B). In 4 animals, NiV genomic RNA and/or antigen were also detected multifocally in neurons in addition to vascular endothelia in brainstems and cerebella (Figure 4, C and D, and Supplemental Table 2), indicating NiV infection spreads faster in these regions. By using immunofluorescence staining and confocal imaging, we could demonstrate that NiV infects both neurons and astrocytes in the brainstem and cerebellum (Figure 4, D-F) during the acute phase of disease. 


\section{A}
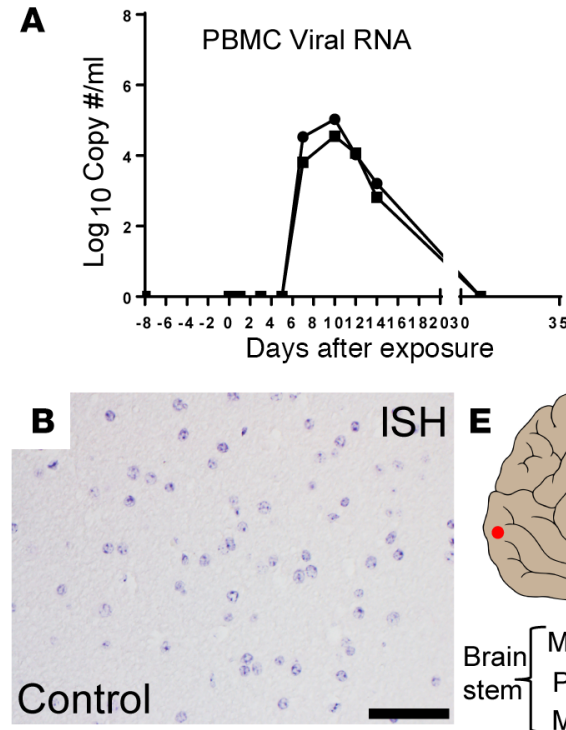

$\mathrm{ISH}$

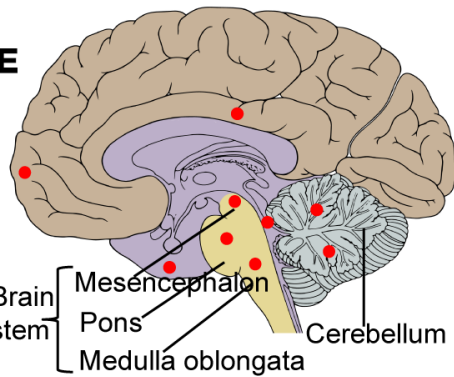

$\rightarrow$ Survivor 1

- Survivor 2
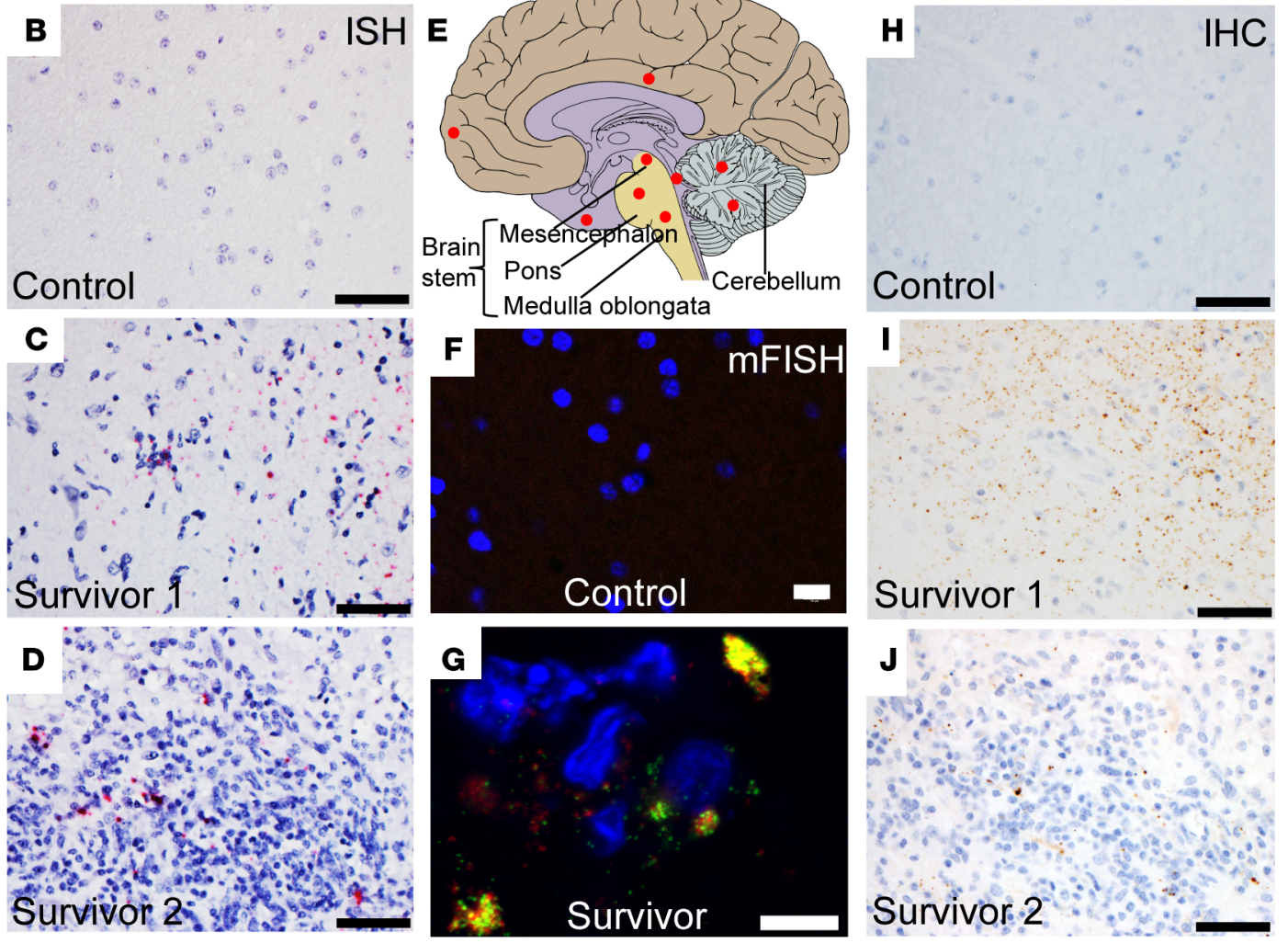

Figure 1. NiV persists in the brains of grivet survivors. (A) NiV genomic RNA in PBMCs of survivor 1 and survivor 2 was determined by reverse transcriptase quantitative PCR (RT-qPCR). No viral RNA was detected in either survivor on study day 32. (B-D) Compared with an uninfected grivet control brain (B), genomic NiV RNA (detected by ISH, red) is present multifocally in hematoxylin-stained (blue) FFPE brain sections (C, cerebral cortex; $\mathbf{D}$, brainstem) of grivet survivors.

(E) Schematic illustration of persistent NiV infection (red dots, viral RNA by ISH) mainly in brainstem and cerebellum in addition to cerebral cortex. (F and $\mathbf{G}$ ) Compared with uninfected grivet control brain (F), both NiV genome (red) and antigenome/mRNA (green) are present in the brain using mFISH (C). Nuclei are stained blue (DAPI). (H-J) Compared with uninfected grivet control brain (H), NiV antigen (IHC, brown) is present in hematoxylin-stained (blue) brain sections of grivet survivors (I and J). Scale bars: $50 \mu \mathrm{m}$ (B-D and $\mathbf{H}-\mathbf{J}), 10 \mu \mathrm{m}$ (F and $\mathbf{G})$.

\section{Discussion}

Here we demonstrate persistent NiV infection in the brain of nonviremic grivet survivors in the absence of infection in any other organs. Based on our findings, NiV persists mainly in neurons and microglial cells (microphages) in the brainstem, cerebral cortex, and cerebellum, accompanied by severe encephalitis. The vascular endothelium is a primary entry route of $\mathrm{NiV}$ into the brain during the acute phase of disease, suggesting that $\mathrm{NiV}$ enters the brain through directly infecting and damaging the blood-brain barrier during acute infection. NiV infection spreads faster in brainstem and cerebellum than other brain regions.

The speculation that the development of relapsing and late-onset encephalitis in humans after initial acute disease is due to persistent $\mathrm{NiV}$ (28) is quite consistent with our observations in grivet survivors. Additionally, NiV genomic RNA was detected in the semen of a survivor on days 16 (Ct value of 36) and 26 (Ct value of 32) but not on days 42 and 59 after onset of illness. Virus isolation was not attempted (9). 

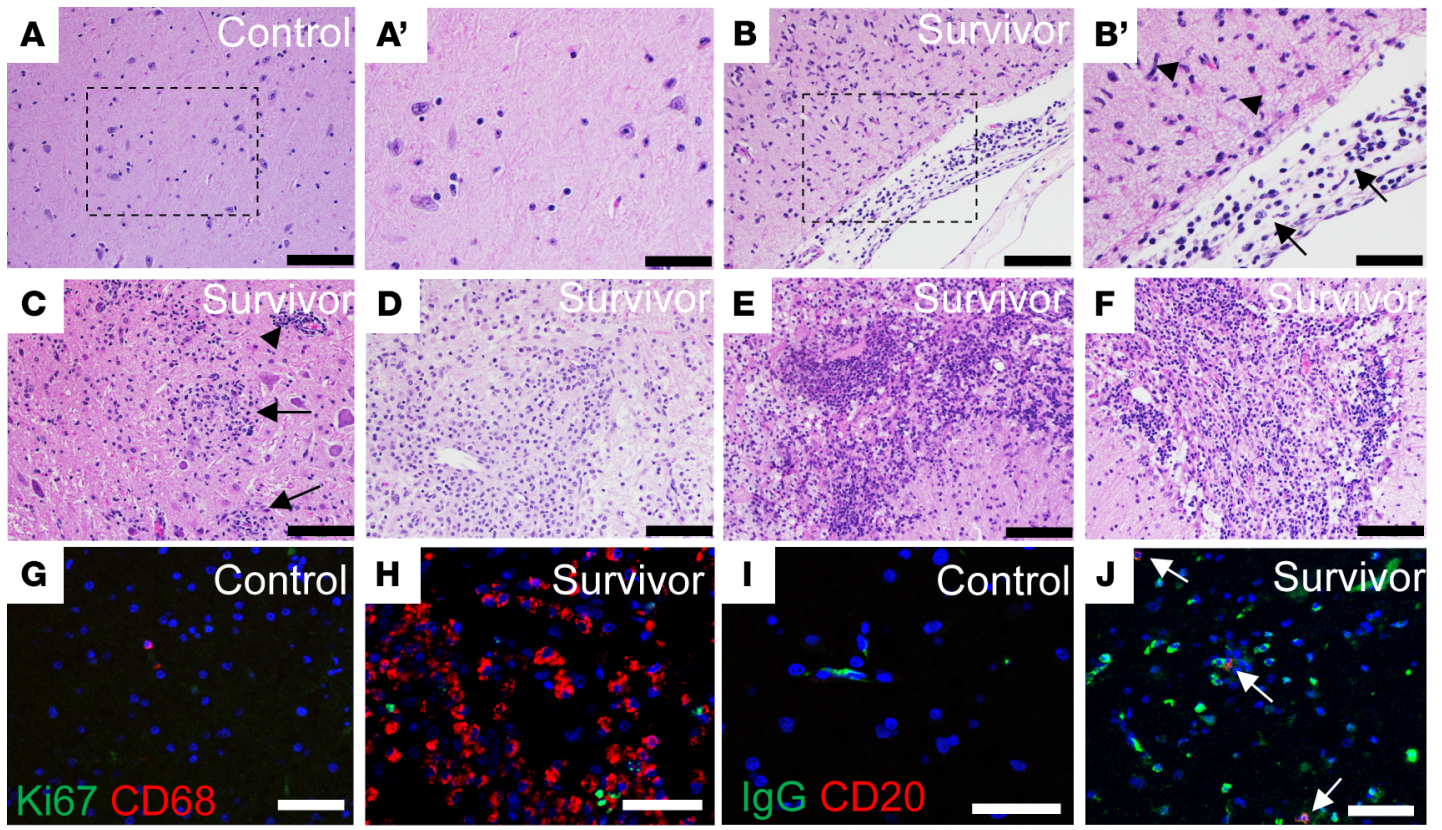

Figure 2. Severe encephalitis in grivet survivors persistently infected with NiV. (A) Normal histologic structure of an uninfected grivet brain. $\mathbf{A}^{\prime}$ is an inset of $\mathbf{A}$ at high magnification. (B) Meningitis (arrow) and microgliosis (arrowhead) in the cerebral cortex of NiV-infected survivors. $\mathbf{B}^{\prime}$ is an inset of $\mathbf{B}$ at high magnification. (C) Glial nodules (arrow) and perivascular infiltration of lymphocytes (arrowhead) in the cerebral cortex of grivet survivors. (D) Neuron depletion and lymphohistiocytic inflammation in the brainstem. (E) Infiltration of inflammatory cells in the white matter and molecular layer of the cerebellum. (F) Granular layer cell depletion and infiltration of inflammatory cells in the cerebellum. Blue, hematoxylin-stained nuclei. (G and $\mathbf{H}$ ) Compared with uninfected grivet brain (G), CD68 ${ }^{+}$microglia (resident macrophages) and $\mathrm{Ki} \mathrm{7}^{+}$cells infiltrate the brain of grivet survivors (H). (I and J) Compared with control uninfected grivet brain (I), IgG antibodies (green) accumulate, and $\mathrm{CD}^{2} \mathrm{O}^{+} \mathrm{B}$ cells (red, indicated by white arrow) infiltrate the brain of grivet survivors (J). Nuclei are stained blue (DAPI). Scale bars: $100 \mu \mathrm{m}(\mathbf{A}, \mathbf{B}$, and $\mathbf{C}-\mathbf{F})$ and $50 \mu \mathrm{m}\left(\mathbf{A}^{\prime}, \mathbf{B}^{\prime}\right.$, and $\left.\mathbf{G}-\mathbf{J}\right)$.

However, we did not observe any viral infection or residual tissue damage in the testes of grivet survivors. The case fatality rate of relapsed and late-onset NiV-associated encephalitis in humans is estimated to be approximately $18 \%$ (28). However, the grivet survivors of our study had no apparent clinical signs of relapsing encephalitis, although encephalitis was noted histopathologically. Perhaps these animals were still developing relapsing encephalitis and, therefore, would have developed clinical disease had the animals lived beyond the preplanned study end date (21). The accumulated histopathologic evidence does not suffice to distinguish between relapsed or late-onset encephalitis and a normal response during convalescence. Additionally, we could not determine whether the observed encephalitis was caused directly by persistent infection of NiV or was a remnant of acute disease in still-recovering animals. It is also possible that both viral persistence and damage from acute-phase disease contributed to the encephalitis. Although performing studies in the Biosafety Level 4 space is logistically and financially challenging, longitudinal studies will be necessary to further dissect the pathogenesis of persistent NiV infection in NHP models.

The cerebella and brainstems of grivet survivors were most strongly affected by encephalitis. Consistent with this finding, many NiV patients have cerebellar dysfunction and prominent signs of brainstem dysfunction, including abnormal vestibuloocular reflex, miosis with variable reactivity, and prominent vasomotor changes resulting in hypertension and tachycardia (15). We found multifocal neuronal NiV infection in the brainstem and cerebellum in addition to vascular endothelial cells, whereas NiV targets only vascular endothelial cells in other brain regions during the acute phase of $\mathrm{NiV}$ disease in grivets. This distribution suggests $\mathrm{NiV}$ infection may spread faster in the cerebellum and brainstem compared with other regions of the brain. $\mathrm{NiV}$ persisted mainly in neurons and microglial cells in the brains of grivet survivors after the virus was cleared from all other organs. In humans, $\mathrm{NiV}$ was also detected mainly in neurons in the brain but also in glial and ependymal cells in cases of fatal late-onset encephalitis (28). Although we could not exclude the possibility that sex might play a role in developing persistent $\mathrm{NiV}$ infection because all the grivets we investigated here were male, NiV persistence and late-onset and relapsing encephalitis have been reported in humans of both sexes. 

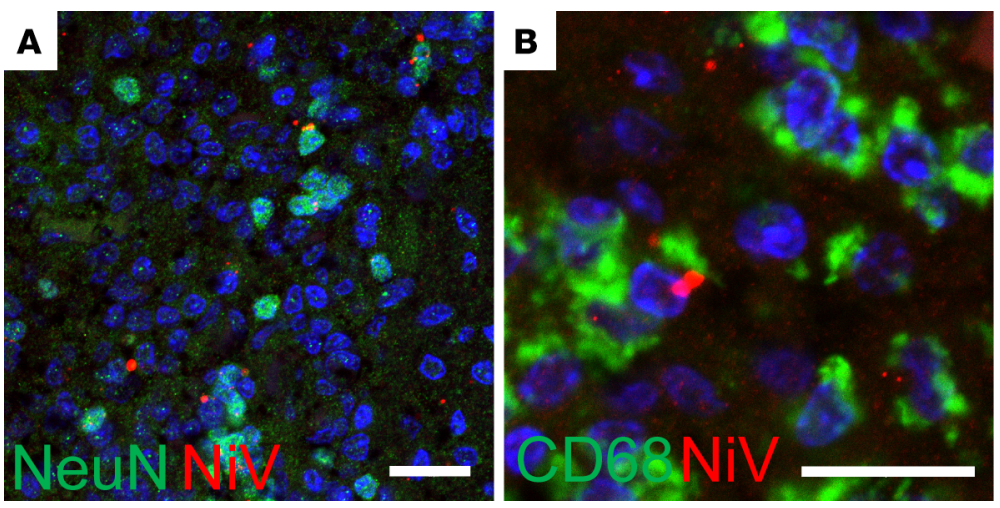

Figure 3. NiV persists mainly in brain neurons and microglia. (A) NiV (red) is present in $\mathrm{NeuN}^{+}$(green) neurons of grivet survivors. (B) NiV (red) in CD68+ (green) microglia in the brains of grivet survivors. Nuclei are stained blue (DAPI). Scale bar: $20 \mu \mathrm{m}$.

Mononegaviruses other than NiV, including measles virus (Paramyxoviridae: Morbillivirus) and Ebola virus (EBOV) and Marburg virus (MARV) (Filoviridae), may persist in immune-privileged sites following acute recovery. Like NiV, measles virus also persists in neurons and glial cells. A serious consequence of this persistence in humans is almost uniformly lethal subacute sclerosing panencephalitis years after recovery from measles (29). We previously reported EBOV and MARV persistence predominantly in the eyes and testes of NHP survivors $(30,31)$. These examples demonstrate that distinct viruses may have different preferences for persistence sites. We consider viral factors and specific host immune environments to orchestrate such viral persistence in different immune-privileged sites.

The data presented here suggest persistent NiV infection may be the main cause of relapsing and late-onset encephalitis, and patients who survive henipavirus infection should be closely monitored for extended periods.

\section{Methods}

Animals. Grivets (Chlorocebus aethiops Linnaeus 1758) were experimentally infected i.t. with $2.5 \times 10^{4}$ (21) or $2 \times 10^{5}$ PFU of the Malaysian strain of NiV, which was isolated from a patient from a 1998 1999 NiV outbreak in Malaysia. A total of 5 pieces of tissue from various brain regions (frontal cortex, corpus striatum, thalamus, midbrain, pons and cerebellum, and medulla oblongata) were collected, FFPE, and retrieved from the United States Army Medical Research Institute of Infectious Diseases (USAMRIID) Pathology Division tissue archives. For histologic evaluation, sections were stained with $H \& E$ after deparaffinization.

$R T-q P C R$. NiV RT-qPCR was performed according to the method reported previously (21). Briefly, cDNA was synthesized by using total RNA extracted from PBMCs with Superscript II and random hexamer priming (Thermo Fisher Scientific). Real-time PCR was performed on a Step One Plus sequence detector (Thermo Fisher Scientific) using TaqMan Universal PCR Master Mix (Thermo Fisher Scientific). Target copy numbers calculated from the $\mathrm{Ct}$ value obtained for each sample were compared using the standard curve equation.

ISH. To detect NiV genomic RNA in FFPE tissues, ISH was performed using the RNAscope 2.5 HD RED kit (Advanced Cell Diagnostics) according to the manufacturer's instructions. Briefly, an ISH probe targeting the NiV genome (nucleotides 2400-4400, GenBank JN808857.1) was designed and synthesized by Advanced Cell Diagnostics (catalog 520071). Tissue sections underwent deparaffinization with Xyless II (Val Tech Diagnostics, LabChem Inc.) and a series of ethanol washes and peroxidase blocking. The sections were then heated in kit-provided antigen retrieval buffer and digested by kit-provided proteinase. Sections were exposed to ISH target probe pairs and incubated at $40^{\circ} \mathrm{C}$ in a hybridization oven for 2 hours. After rinsing with wash buffer, ISH signal was amplified using kit-provided Pre-amplifier and Amplifier conjugated to alkaline phosphatase and incubated with Fast Red substrate solution (Advanced Cell Diagnostics) for 10 minutes at room temperature. Sections were then stained with hematoxylin (Poly Scientific R\&D Corp), air-dried, and mounted. An additional section from each tissue block was examined when the first section was ISH negative. 

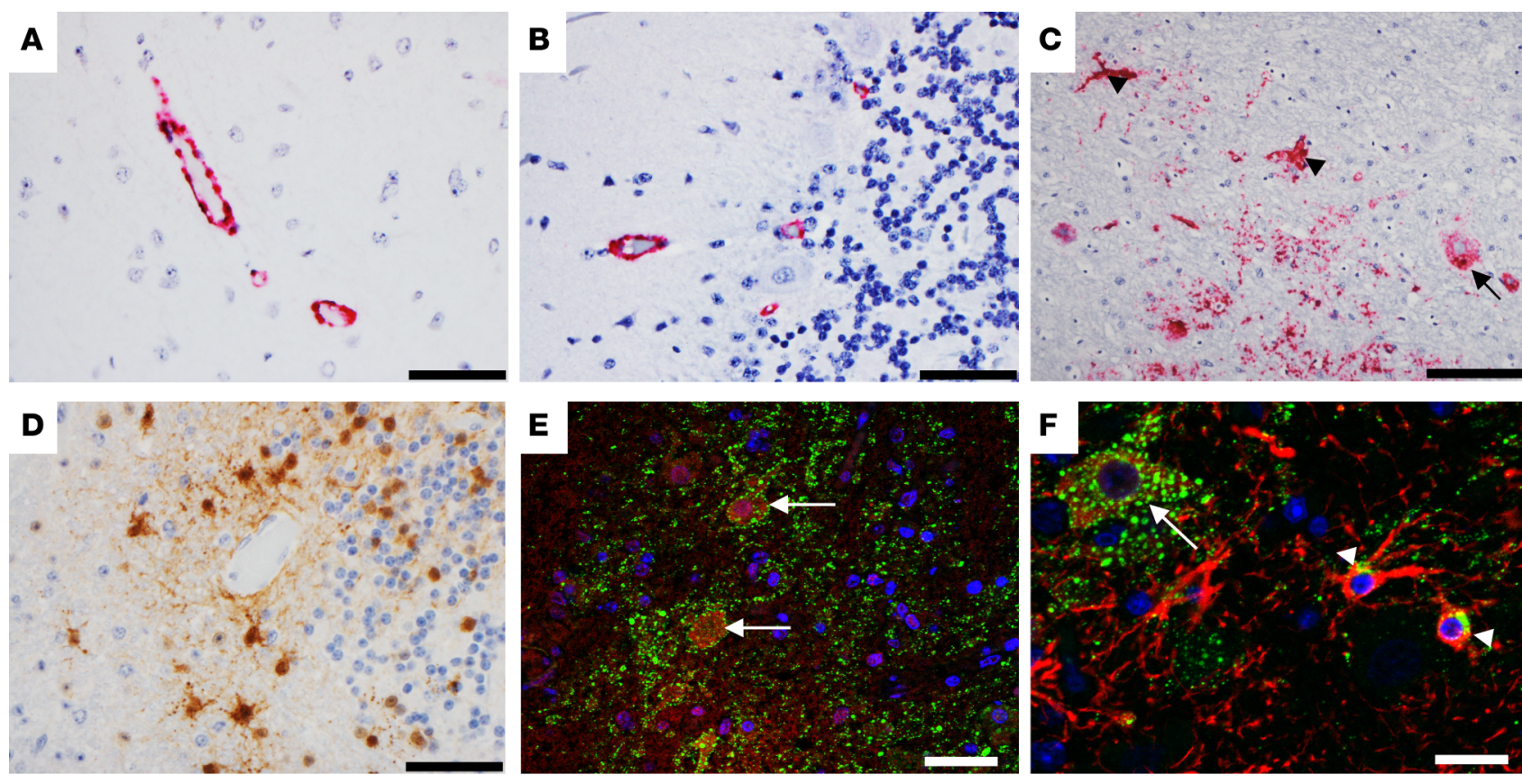

Figure 4. NiV brain persistence is established through infection of the vascular endothelium. (A-C) NiV (ISH, red) infection in the vascular endothelium of the cerebrum (A), cerebellum (B), and brainstem (C) in an animal that died 9 days after exposure. (C) Arrow, NiV-infected neuron; arrowhead, NiV-infected vascular endothelium. (D) Massive NiV infection in the cerebellum (brown, IHC) in an animal that died 10 days after exposure. Blue, hematoxylin-stained nuclei. (E) NiV (green) infection in $\mathrm{NeuN}^{+}$(red, arrow) neurons and (F) Glial fibrillary acidic protein-positive (GFAP) (red, arrowhead) astrocytes in the brain of an animal that died 10 days after exposure. Arrow, NiV-infected neuron. Blue, nuclear stain (DAPI). Scale bars: $50 \mu \mathrm{m}$ (A-D) and $20 \mu \mathrm{m}$ (E and F).

mFISH was performed using the RNAscope Fluorescent Multiplex Kit (Advanced Cell Diagnostics) according to the method reported previously (32). Thirty ZZ probe pairs optimized to $\mathrm{C} 1 \mathrm{channel}$ (red, catalog 520071) targeting the NiV genome (nucleotides 2400-4400, GenBank JN808857.1) were designed and synthesized by Advanced Cell Diagnostics (catalog 520071), and $40 \mathrm{ZZ}$ probe pairs optimized to C3 channel (catalog 520081-C3, green) targeting the NiV antigenome (nucleotides 8941-11,160, GenBank JN808857.1) were synthesized by Advanced Cell Diagnostics. FFPE tissue sections underwent deparaffinization with Xyless II, a series of ethanol washes, and treatment with $0.1 \%$ Sudan Black B (MilliporeSigma) to reduce autofluorescence. Tissues were heated in kit-provided antigen retrieval buffer and digested by kit-provided proteinase. Sections were exposed to $\mathrm{mFISH}$ target probe pairs and incubated at $40^{\circ} \mathrm{C}$ in a hybridization oven for 2 hours. After rinsing with washing buffer, mFISH signal was amplified using company-provided Pre-amplifier and Amplifier conjugated to fluorescent dye Alexa Fluor 488 or Atto 550 provided in kit. Sections were counterstained with DAPI (Thermo Fisher Scientific), mounted, and stored at $4^{\circ} \mathrm{C}$ until image analysis. mFISH images were captured on an LSM 880 confocal microscope (Zeiss) and processed using open-source ImageJ software (NIH).

IHC. ISH-positive slides were further examined by IHC. IHC was performed using the Dako Envision system (Dako Agilent Pathology Solutions). After deparaffinization, rehydration, and methanol/hydrogen peroxide blocking, slides were stained using a rabbit polyclonal anti-NiV Ab (USAMRIID, 1294) at a dilution of 1:4000 (21), followed by an HRP-conjugated, secondary anti-rabbit polymer (Dako Agilent Pathology Solutions, K4003). All slides were exposed to brown chromogenic substrate DAB (Dako Agilent Pathology Solutions, K3468), counterstained with hematoxylin, dehydrated, and coverslipped.

Immunofluorescence staining. After deparaffinization and reduction of autofluorescence, tissues were heated in citrate buffer, pH 6.0 (MilliporeSigma), for 15 minutes to reverse formaldehyde cross-links. After rinsing with PBS, pH 7.4 (Thermo Fisher Scientific), sections were blocked overnight with PBS containing $5 \%$ normal goat serum (MilliporeSigma) at $4^{\circ} \mathrm{C}$. Sections were then incubated with the following primary Abs for 2 hours at room temperature: rabbit polyclonal Ab against NiV (USAMRIID, 1294) at a dilution of 1:1000 (21); rabbit polyclonal $\mathrm{Ab}$ against Ki67 at a dilution of 1:400 (ab15580, Abcam); mouse antihuman CD20 Ab at a dilution of 1:200 (ab9475, Abcam); chicken polyclonal anti-NeuN Ab at a dilution 
of 1:100 (ab134014, Abcam); chicken polyclonal anti-GFAP Ab at a dilution of 1:1000 (ab4674, Abcam); mouse anti-human CD68 Ab at a dilution of 1:200 (clone KP1, Dako Agilent Pathology Solutions); or rabbit polyclonal anti-human IgG at a dilution of 1:100 (F020202-2, Dako Agilent Pathology Solutions). After rinsing in PBS, sections were incubated with secondary goat IgG Alexa Fluor 488-conjugated anti-rabbit, anti-mouse, or anti-chicken Abs and with goat IgG Alexa Fluor 561-conjugated anti-mouse or anti-rabbit Abs (Life Technologies) for 1 hour at room temperature. Sections were coverslipped using VECTASHIELD antifade mounting medium with DAPI (Vector Laboratories). Images were captured and processed as described above.

Study approval. All animal studies described or referred to in this manuscript were conducted under USAMRIID IACUC-approved protocols (AP-11-006, Frederick, Maryland, USA) in compliance with the Animal Welfare Act, Public Health Service Policy, and other federal statutes and regulations relating to animals and experiments involving animals. The facility in which this research was conducted is accredited by the Association for Assessment and Accreditation of Laboratory Animal Care, International, and adheres to principles stated in the Guide for the Care and Use of Laboratory Animals (National Academies Press, 2011).

\section{Author contributions}

$\mathrm{XZ}$ conceived and designed the experiments. JL, KMC, SCJ, AMB, TMB, SYL, ANH, and XZ performed experiments. JHK and $\mathrm{XZ}$ interpreted the data and wrote the manuscript with input from all authors.

\section{Acknowledgments}

We thank Laura Bollinger (NIH/National Institute of Allergy and Infectious Diseases [NIAID] Integrated Research Facility at Fort Detrick, Frederick, Maryland, USA) for critically editing the manuscript. We thank William Discher (USAMRIID, Frederick, Maryland, USA) for the brain diagram and Jennifer Chapman at USAMRIID for critically reviewing the manuscript draft. We also thank Thomas Briese and Ian Lipkin (Columbia University, New York, New York, USA); Joshua Shamblin, Heather Esham, Ginger Donnelly, Joshua Johnson, and William Pratt (USAMRIID); and Lisa Hensley (NIH/NIAID Integrated Research Facility at Fort Detrick) for their contributions to the original animal studies. JHK's participation was funded, in part, through Battelle Memorial Institute's prime contract with the US NIAID under contract HHSN272200700016I (to JHK). The views and conclusions contained in this document are those of the authors and should not be interpreted as necessarily representing the official policies, either expressed or implied, of the US Department of the Army, the US Department of Defense, the US Department of Health and Human Services, or of the institutions and companies affiliated with the authors. In no event shall any of these entities have any responsibility or liability for any use, misuse, inability to use, or reliance upon the information contained herein. The US departments do not endorse any products or commercial services mentioned in this publication.

Address correspondence to: Xiankun (Kevin) Zeng, United States Army Medical Research Institute of Infectious Diseases (USAMRIID), 1425 Porter St., Fort Detrick, Frederick, Maryland 21702, USA. Phone: 301.619.3401; Email: xiankun.zeng.fn@mail.mil.

ANH's present address is: Vaccine Research Center, National Institute of Allergy and Infectious Diseases, NIH, Bethesda, Maryland, USA.

1. Amarasinghe GK, et al. Taxonomy of the order Mononegavirales: update 2019. Arch Virol. 2019;164(7):1967-1980.

2. World Health Organization. ICD-11 for Mortality and Morbidity Statistics (December 2018). WHO Web Site. https://icd.who. int/browse11/1-m/en\#/http://id.who.int/icd/entity/1020283662. Updated April 2019, accessed on April 3, 2019.

3. Ang BSP, Lim TCC, Wang L. Nipah virus infection. J Clin Microbiol. 2018;56(6):e01875-17.

4. Chua KB, et al. Nipah virus: a recently emergent deadly paramyxovirus. Science. 2000;288(5470):1432-1435.

5. Weatherman S, Feldmann H, de Wit E. Transmission of henipaviruses. Curr Opin Virol. 2018;28:7-11.

6. Chua KB, et al. Isolation of Nipah virus from Malaysian Island flying-foxes. Microbes Infect. 2002;4(2):145-151.

7. Hsu VP, et al. Nipah virus encephalitis reemergence, Bangladesh. Emerg Infect Dis. 2004;10(12):2082-2087.

8. Spiropoulou CF. Nipah virus outbreaks: still small but extremely lethal. J Infect Dis. 2019;219(12):1855-1857.

9. Arunkumar G, et al. Persistence of Nipah virus RNA in semen of survivor [published online ahead of print December 22, 2018]. Clin Infect Dis. 2019;69:377-378.

10. Rahman MA, et al. Date palm sap linked to Nipah virus outbreak in Bangladesh, 2008. Vector Borne Zoonotic Dis. 2012;12(1):65-72.

11. Gurley ES, et al. Person-to-person transmission of Nipah virus in a Bangladeshi community. Emerging Infect Dis. 
2007;13(7):1031-1037.

12. Tan CT, Tan KS. Nosocomial transmissibility of Nipah virus. J Infect Dis. 2001;184(10):1367.

13. Mounts AW, et al. A cohort study of health care workers to assess nosocomial transmissibility of Nipah virus, Malaysia, 1999. J Infect Dis. 2001;183(5):810-813.

14. Chua KB, et al. Fatal encephalitis due to Nipah virus among pig-farmers in Malaysia. Lancet. 1999;354(9186):1257-1259.

15. Goh KJ, et al. Clinical features of Nipah virus encephalitis among pig farmers in Malaysia. N Engl J Med. 2000;342(17):1229-1235

16. Mungall BA, et al. Vertical transmission and fetal replication of Nipah virus in an experimentally infected cat. J Infect Dis. 2007;196(6):812-816.

17. Torres-Velez FJ, et al. Histopathologic and immunohistochemical characterization of Nipah virus infection in the guinea pig. Vet Pathol. 2008;45(4):576-585.

18. Clayton BA, et al. Transmission routes for Nipah virus from Malaysia and Bangladesh. Emerging Infect Dis. 2012;18(12):1983-1993.

19. Wong KT, et al. A golden hamster model for human acute Nipah virus infection. Am J Pathol. 2003;163(5):2127-2137.

20. Geisbert TW, et al. Development of an acute and highly pathogenic nonhuman primate model of Nipah virus infection. PLoS One. 2010;5(5):e10690

21. Johnston SC, et al. Detailed analysis of the African green monkey model of Nipah virus disease. PLoS One. 2015;10(2):e0117817.

22. Bossart KN, et al. A neutralizing human monoclonal antibody protects against lethal disease in a new ferret model of acute Nipah virus infection. PLoS Pathog. 2009;5(10):e1000642

23. Hammoud DA, et al. Aerosol exposure to intermediate size Nipah virus particles induces neurological disease in African green monkeys. PLoS Negl Trop Dis. 2018;12(11):e0006978.

24. Cong Y, et al. Loss in lung volume and changes in the immune response demonstrate disease progression in African green monkeys infected by small-particle aerosol and intratracheal exposure to Nipah virus. PLoS Negl Trop Dis. 2017;11(4):e0005532.

25. Middleton DJ, et al. Experimental Nipah virus infection in pigs and cats. J Comp Pathol. 2002;126(2-3):124-136.

26. Dhondt KP, et al. Type I interferon signaling protects mice from lethal henipavirus infection. J Infect Dis. 2013;207(1):142-151.

27. Dups J, Middleton D, Long F, Arkinstall R, Marsh GA, Wang LF. Subclinical infection without encephalitis in mice following intranasal exposure to Nipah virus-Malaysia and Nipah virus-Bangladesh. Virol J. 2014;11:102.

28. Tan CT, et al. Relapsed and late-onset Nipah encephalitis. Ann Neurol. 2002;51(6):703-708.

29. Griffin DE, Lin WH, Pan CH. Measles virus, immune control, and persistence. FEMS Microbiol Rev. 2012;36(3):649-662.

30. Coffin KM, et al. Persistent Marburg virus infection in the testes of nonhuman primate survivors. Cell Host Microbe. 2018;24(3):405-416.e3.

31. Zeng X, et al. Identification and pathological characterization of persistent asymptomatic Ebola virus infection in rhesus monkeys. Nat Microbiol. 2017;2:17113.

32. Liu J, et al. A novel sheet-like virus particle array is a hallmark of Zika virus infection. Emerg Microbes Infect. 2018;7(1):69. 